\title{
Conexões
}

Educação Fisica, Esporte e Saúde

\section{Entrevista a Francisco Lagardera, referente intelectual de la educación física y de la ciencia de la acción motriz}

Unai Saez de Ocariz Granja ${ }^{1}$

Pere Lavega-Burgués²

\section{RESUMEN}

En el presente escrito se expresan las reflexiones compartidas por Francisco Lagardera, Catedrático del Instituto Nacional de Educación Física de Cataluña (INEFC), Licenciado en Historia, profesor del INEFC durante más de 30 años y maestro de Educación Física desde hace 45 años. En la conversación mantenida con el Dr. Lavega en un contexto muy cordial, el profesor Lagardera hace un recorrido en su historia académica desde que finalizó sus estudios universitarios hasta la actualidad. Muestra el significado que ha tenido para su vida profesional y científica la Ciencia de la Acción Motriz o Praxiología Motriz. Comparte una visión muy personal de algunas de las vivencias que marcaron su trayectoria, subrayando aquellos hitos que, bajo su percepción, supusieron un punto de inflexión tanto para él como para la Praxiología Motriz.

Palabras Clave: Praxiología motriz. Educación física. Conducta motriz. Ciencia de la acción motriz. Formación universitaria.

\footnotetext{
1 Universidad de Barcelona.

2 Universidad de Lleida.
}

\section{Correspondência:}

Unai Saez de Ocariz Granja. Instituto Nacional de Educación Física de Cataluña (INEFC) de Barcelona, Departament de la Presidència, Generalitat de Catalunya, Av. de I'Estadi 12-22, 08038, Barcelona, España, Email: usaez@gencat.cat 


\section{Entrevista a Francisco Lagardera, referente intelectual da educação física e da ciência da ação motriz}

\section{RESUMO}

Este artigo expressa as reflexões compartilhadas por Francisco Lagardera, professor do Instituto Nacional de Educação Física da Catalunha (INEFC), bacharel em História, professor do INEFC há mais de 30 anos e professor de educação física há 45 anos. Numa conversa com o Dr. Lavega, em um contexto muito cordial, o professor Lagardera traça sua história acadêmica desde o final de seus estudos universitários até os dias atuais. Mostra o significado que a Ciência da Ação Motriz ou a Praxiologia Motriz teve para sua vida profissional e científica. Ele compartilha uma visão muito pessoal de algumas das experiências que marcaram sua carreira, sublinhando os marcos que, em sua percepção, representavam um ponto de virada para ele e a Praxiologia Motriz.

Palavras-chave: Praxiologia motriz. Educação Física. Comportamento motor. Ciência da ação motriz. Formação universitária.

\section{Interview with Francisco Lagardera, intellectual referent of physical education and the science of motor action}

\section{ABSTRACT}

This paper expresses the reflections shared by Francisco Lagardera, Professor at the National Institute of Physical Education of Catalonia (INEFC), Bachelor of History, professor at INEFC for more than 30 years and teacher of Physical Education for 45 years. In a conversation with Dr. Lavega in a very cordial context, Professor Lagardera traces his academic history from the end of his university studies to the present day. It shows the meaning that Motor Action Science or Motor Praxiology has had for his professional and scientific life. He shares a very personal vision of some of the experiences that marked his career, underlining those milestones that, under his perception, represented a turning point for both him and Motor Praxiology.

Keywords: Motor praxiology. Physical education. Motor conduct. Motor action science. University education. 


\section{Pere LAVega:}

Hoy es día 10 de abril de 2020 y estamos dos amigos, Paco y Pere, compartiendo una conversación motivada por aquello que nos ha aportado la ciencia de la acción motriz o praxiología motriz.

Creemos que puede contextualizarse lo que ha supuesto esta disciplina a través de la biografía de Paco, tanto desde el plano intelectual como profesional.

Vamos a tratar diferentes temas. Paco es un hombre de la teoría y es también un hombre de práctica, por ello puede acercarse a esta disciplina desde distintas perspectivas y tiempos de su biografía, desde que la descubre hasta ahora que ha adquirido mucha importancia en su vida cotidiana.

La praxiología motriz es una nueva disciplina que surgió en Francia y llegó en un momento determinado a España. Hacia el año 1984, siendo alumno tuyo de sociología de la actividad física y del deporte, empecé a oír que nos hablabas de esta disciplina: ¿Cómo te llegó este conocimiento?

\section{FRANCISCO LAGARDERA:}

Llegué a Madrid hacia primeros de septiembre 1969 para estudiar educación física. Finalicé los estudios en junio de 1973 y durante esos cuatro años nadie pronunció el nombre de Pierre Parlebas, ni nadie me comentó lo que este profesor había publicado desde el año 1967.

En septiembre de 1973 empecé a trabajar como profesor de educación física y maestro en un colegio público de Barcelona. Cada día cuando finalizaba mis jornadas como docente me decía a mi mismo: iEsto no es educación física, esto que hago no es educación física!

No tenia ni idea de cómo educar a mis alumnos impartiendo clases de educación física ¿Qué aprendían en mis clases? ¿Qué aportaban mis clases hechas en el patio a su educación general? ¿Aprendían algún conocimiento específico y útil?

Mi formación básica consistió en acumular conocimiento técnico de muchas modalidades deportivas. Tan solo disponía como recurso fiable un trabajo mancomunado que habíamos hecho en clase de "juegos tradicionales españoles" recopilados entre todos los alumnos.

Los juegos tradicionales, mi intuición y la creatividad era lo que disponía para reinventarme como profesor en cada clase $y$ ofrecer a los alumnos una experiencia educativa realizada en el patio: jugando, corriendo, saltando, 
compitiendo o cooperando, pero iba sin orientación, sin brújula, no sabía a dónde dirigía a mis alumnos.

En 1978 logré una plaza de profesor en el Servicio de Deportes de la Universidad Autónoma de Barcelona, aunque simultaneaba a la vez ambos trabajos. Al año siguiente, un compañero de la universidad (Antonio Galera), tuvo la iniciativa de traducir algunos artículos publicados en Inglaterra $y$ en Francia, entre ellos tres artículos de Pierre Parlebas: "La sociomotricidad: ¿una nueva forma de abordar la educación física" (1967); "La educación física: una educación de las conductas motrices (1967) y "Estructura y conducta motriz" (1969).

iEstos textos fueron mi auténtico talismán! En ellos encontré el orden que necesitaba, pude orientar mi ejercicio docente, en realidad fueron "mi verdadera universidad para formarme como profesor de educación física", lo anterior fue un pobre bagaje con algún barniz humanista que hallé en las lecturas de J. Ma. Cagigal.

Desde entonces mi vida profesional cambió y también lo hizo mi vida privada. Con este reducido capital parlebasiano, empecé a plantear situaciones motrices muy distintas al modificar aspectos de la lógica interna de un juego o bien de una modalidad deportiva: reduciendo o aumentando espacios, cambiando el material, balones más baratos para cada alumno, estimulé la creatividad de los alumnos..., tanto en las escuelas públicas en donde trabajé, como en la universidad o en el instituto de enseñanza media para motivarlos y participar en el cambio de las normas para practicar una u otra situación motriz que les interesaba.

En todas las instituciones en donde trabajé lo pasaba muy bien, no tenía un trabajo sino un gran divertimiento, disfrutaba con mi trabajo y los alumnos también. La educación física tenía un sentido como educación, eficaz y profunda, una experiencia educativa muy placentera.

A pesar de todo no pude ampliar mi conocimiento sobre el resto de publicaciones de Parlebas, las circunstancias históricas me llevaban como un torbellino semana a semana, con lo que disponía tenía suficiente para justificar mi docencia en educación física, además mi ignorancia en el francés fue un obstáculo añadido.

No llegué a aplicar con claridad la educación de las conductas motrices. Poco antes de convertirme en profesor del INEFC de la universidad de Lleida, aplicaba la autoevaluación en base a indicadores que acumulaba sobre la evolución que ellos tenían de sus conductas motrices. Los alumnos constataban con sus sensaciones, sus intuiciones y la conciencia sus progresos contestando a un cuestionario. En cada nuevo curso lo modificaba en función de los resultados 
del año anterior.

Para la mayoría de mis colegas mi obrar era un camelo, un fraude o peor, una injusticia que los alumnos se autocalificaran. Para mi fue la mejoría educativa que encontré y desarrollé para mis clases, la autoevaluación de las conductas motrices de los alumnos.

Todo lo que hacía como profesor lo alimentaban de este manantial parlebasiano, muy humilde, muy pobre, en mis alforjas intelectuales apenas había tres campanas del campanario de la inmensa catedral que ya era la obra de Parlebas, construida y publicada.

Con este pobre bagaje, repleto de ignorancia por la obra casi completa en 1985, me presenté a las oposiciones de profesor de enseñanza medias en Madrid y profesor en el INEFC de la universidad de Lleida, en todas ellas obtuve resultados positivos, presentándome, escribiendo y expresándome con el salva conducto de la obra de Parlebas.

Al año siguiente, noviembre de 1986, el compañero, Domingo Blázquez invitó a Parlebas a presentar en el INEFC de Barcelona su libro "Elements de sociologie du sport", recien publicado en Presses Universitaires de France (Paris, PUF). Allí conocí personalmente a quién, hacía ya siete años, era mi auténtico maestro y genio de la nueva educación física, de la educación de las conductas motrices.

iFue una bomba afectiva e intelectual asistir a sus enseñanzas en directo!

El mundo de la educación física, desde ese momento, acababa de dar para mi un giro copernicano, en especial cuando observé las reacciones de gran parte del profesorado que asistieron a estas jornadas, quienes se aferraban al paradigma clásico del movimiento, que seguía vigente desde el siglo XVIII.

En ese momento me di cuenta de que no me había convertido en un profesor elegido para ejercer en la catedral de la educación física en España, los INEFC, pues se convirtieron ante mis ojos en escuelas de deporte, no en instituciones universitarias, que es lo que esperaba encontrar en ellas.

\section{Pere lavega:}

Recuerdo este momento, porque fui alumno en el INEFC de Lleida de la segunda promoción en 1983, también recuerdo estos apuntes utilizados por ti en clase. Unos pocos años más adelante, en torno 1990, tomaste una decisión crucial, la de formar en septiembre un "grupo de estudios e investigaciones en praxiología motriz. Este grupo estaba compuesto por profesores jóvenes 
(exalumnos) entre los que me encontraba, por ello me siento orgulloso, de haber tenido el privilegio de ser una de las personas que iniciamos esta aventura.

Explica qué dinámicas se activaron en este grupo y qué supuso para ti esta experiencia académica en tu trayectoria.

\section{FRANCISCO LAGARDERA:}

Esta iniciativa suponía poner una primera piedra para organizarnos como en la universidad: estudiar, investigar, debatir, escribir, publicar, compartir lo que hacíamos entre todos los que formábamos este grupo. También participar en eventos $y$ foros para compartir experiencias con otros investigadores $y$ profesores..., era un quehacer usual en la universidad. Trabajábamos en equipo, cooperando, sin competir, ayudándonos entre todos. Las personas que se sumaban aceptaban libremente la dinámica que se generaba en el grupo, sintiéndonos libres para compartir nuestras inquietudes, nuestros sueños $y$ nuestros trabajos. Todo se discutía, se reflexionaba, se revisaba...

Una aventura maravillosa de trabajo en equipo que ha dado frutos año a año, poco a poco, lentamente, tal y como se genera el conocimiento, así es el proceder de la ciencia, con la certeza de que esta dinámica, más pronto que tarde abriría una perspectiva de avance en lo que era nuestro propósito central: consolidar el objeto pertinente de la educación física, educar e influir positivamente en las conductas motrices de nuestros alumnos, optimizándolas entre atletas y clientes, siempre apoyados en los fundamentos de la teoría de la acción motriz que generaba una región de conocimiento especial y autónoma de la práctica motriz; que además, nos permitía investigar aplicando métodos y preceptos científicos que nos aportaba la praxiología motriz.

Fueron años con mucho entusiasmo, pero también con precipitación y falta de experiencia para publicar, presentando comunicaciones en eventos científicos que no tenían el rigor que hubiésemos pretendido.

\section{Pere LAVeGA:}

En esta época, la recuerdo muy bien, este grupo fue evolucionando hasta que en el lunes 16 de octubre de 1995 defendí mi tesis doctoral, la primera que se fundamentaba desde la praxiología motriz en España. Parlebas fue miembro del tribunal, junto a José Hernández profesor en la facultad de Educación Física de Las Palmas, Carles Feixa, antropólogo de la UDL y Antoni Petrus como representante del departamento de Teoría e Historia de la Educación en la universidad de Barcelona. Se estableció a la vez un punto de inflexión con la organización del primer seminario internacional de praxiología motriz que se 
organizó en el INEFC de Lleida al día siguiente.

Una segunda etapa en la que se generó una red internacional de colegas, profesores e investigadores. ¿Cómo viviste esta época?

\section{FRANCISCO LAGARDERA:}

Tu tesis fue un hecho histórico, hubo un antes y un después de este acontecimiento. Fue la primera tesis doctoral fundamentada en la praxiología motriz, elaborada por un miembro fundador del grupo, además fue la primera tesis que dirigí, cuyos procesos metodológicos que se utilizaron se compartieron en el grupo. Era también la tesis del doctor Pere Lavega, pero también fue del grupo de estudios e investigaciones en praxiologia motriz de Lleida.

Parlebas formó parte del tribunal, asistieron colegas de otros centros españoles, colegas franceses como During o Collard, tunecinos, portugueses, personas que venían a Lleida para participar en el Primer Seminario Internacional de Praxiología Motriz. Cuando finalizó ese seminario, se inició al día siguiente un Congreso que organizaba el INEFC en el que aparecía, por primera vez, una sección dedicada a las ponencias y comunicaciones sobre praxiología motriz.

En esa semana el grupo de investigación cumplía su primer lustro y alcanzábamos la mayoría edad. Pasamos a crear una red internacional para compartir nuestros pensamientos, proyectos, publicaciones $y$ de las investigaciones.

El grupo se fue nutriendo de personas cada vez más interesadas, no tan solo del centro de Lleida, sino que acudían estudiantes desde Barcelona, Zaragoza, Girona, Castellón, de Francia y hasta desde Brasil y Chile para estudiar e investigar con la praxiología.

Se iniciaron proyectos de tesis doctorales sobre el parapente, la gimnasia, el cicloturismo, la expresión motriz, experiencias docentes en el medio natural o hasta prácticas motrices introyectivas..., estábamos abiertos a cualquier pretensión de quienes deseaban compartir nuestra dinámica.

Sin embargo, pese a nuestro interés para iniciar investigaciones sobre educación de las conductas motrices, nadie aceptaba nuestra invitación. Este seguía siendo el lado oculto de la investigación praxiológica, de las conductas motrices.

Sabedores de esta necesidad para llegar hasta la necesidad cotidiana de los profesores de educación física que ejercían en miles de escuelas, institutos y liceos del mundo, decidimos investigar sobre las conductas motrices de nuestros 
alumnos, tu en la asignatura de juegos tradicionales y yo en las prácticas motrices introyectivas. Y hasta aquí hemos llegamos, con algunos artículos, algún libro, algunas ponencias... y en este lugar nos quedamos, sin tocar el hueso ni el músculo de esta nueva pedagogía que podría revolucionar la educación mundial.

Pero a pesar de nuestras limitaciones, tu tesis doctoral fue un hito $y$ también iniciar en Lleida un ciclo muy productivo de seminarios internacionales que nos propulsó hacia el futuro con mucha esperanza.

\section{Pere LAVegA:}

Efectivamente, ese día la sala estaba repleta de colegas que acudían de otros centros de España, desde Francia como Bertrand During y Luc Collard o colegas como Elloumi que asistieron desde Túnez ya que, al día siguiente, martes 18 de octubre de 1995 iniciamos otra etapa crucial, el primer seminario internacional de praxiología motriz, y el miércoles 19 de octubre se inició un Congreso en el INEFC de Lleida en el que por primera vez la praxiología motriz contaba como una sección específica.

Desde este momento se sucedieron sucesivos eventos y seminarios cada dos años, en Paris, Amiens, Las Palmas, Barcelona, La Coruña y Madrid, en este encuentro, el de la capital de España, hubo cosas muy importantes que cambiaron de nuevo esta maravillosa aventura de la praxiología motriz.

¿Qué pasó en una estación de trenes madrileña?

\section{FRANCISCO LAGARDERA:}

En primer lugar, fue importante este foro porque Pere y Paco presentamos en el INEF de Madrid el "nuevo léxico de praxiología motriz" que revisamos y prologamos. Era un gran sueño, poder disponer de la obra básica de Parlebas en español.

Sabíamos que esta red científica y teórica aún era tierna, incluso verde. Se presentaban muchos trabajos que no tocaban ni la piel de la disciplina, ni tan solo dándole caricias. La sombra del paradigma clásico seguía acechando.

Paseando por Atocha para regresar a Lleida apareció entre nosotros la ilusión de hacer posible que la universidad de Lleida pudiese otorgar la investidura a Pierre Parlebas como doctor honoris causa.

Se trataba de un académico y científico de talla mundial que había creado 
una pedagogía, la educación de las conductas motrices, que centraba el interés pedagógico en la persona para influir en sus conductas motrices. Un hallazgo sin parangón; creando, además, un espacio epistemológico específico y pertinente para la motricidad humana, el área de conocimiento de la acción motriz desarrollando la teoría de la acción motriz. Un corpus de saber que no existía hasta su aparición con sus primeras publicaciones desde 1967 hasta esa fecha, el año 2001 en que apareció la publicación española del nuevo léxico. Finalmente, como un genio que fue capaz de construir una nueva ciencia, no por casualidad, sino cubriendo una necesidad, la laguna que tenía la ciencia con la motricidad humana sin especificidad, por esto tendría que pedir cobijo en las ciencias de la vida, en las ciencias formales o como de las ciencias humanas.

A partir de tu tesis y de la mano de Carles Feixa, participamos en seminarios sobre la vida cotidiana con Llorens Prats y Roberto Fernández, hasta hace poco rector de la Universidad de Lleida. Colegas que cada vez apreciaban más y mejor nuestras aportaciones desde la educación física, desde los juegos tradicionales y desde los deportes con la praxiología motriz.

Ya estábamos trabajando con la universidad, codo a codo, por esto nuestro sueño podía ser factible gracias a nuestro trabajo, a nuestras influencias y sobre todo por la relevancia del personaje que propusimos.

\section{Pere LAVegA:}

El año 2002 fue un año realmente muy especial para la ciencia de la acción motriz. Pierre Parlebas fue investido doctor honoris causa por la Universidad de Lleida, en España ¿Cómo viviste este hito?

\section{FRANCISCO LAGARDERA:}

En octubre de 2002 Pierre Parlebas fue investido doctor honoris causa. Con este acto el INEFC de Lleida entró por la puerta grande en la universidad, aunque no era aún una facultad, pero sí ya era universitario su proceder.

Para la educación física del mundo, para la praxiología motriz y para lo obra inmensa de Parlebas, este reconocimiento supuso ya la mayoría internacional de nuestra comunidad científica. Aquí, en España, en Francia, en Europa, en África y en América.

Muchos colegas que fui encontrando en diversos eventos, irreductibles defensores del paradigma del decimoctavo siglo, el movimiento, todos nos felicitaban por este hito de la educación física, para aquí y para todo el resto del mundo. 


\section{Pere lavega:}

Además, fue la primera vez que en España se dio este reconocimiento a un profesor y un científico que procedía del área de la educación física, merecerlo por ser científico de la motricidad, creador del área de conocimiento de la acción motriz...

\section{FRANCISCO LAGARDERA:}

Y también internacionalmente porque la obra de Parlebas es tan grande, tan monumental, que rebasa con mucho las fronteras de los países, es una aportación importantísima al acervo de la civilización humana. Supuso un gran salto. A partir de este momento esta historia es mucho más conocida, más internacional.

En 2010 el seminario internacional de Praxiología Motriz se celebró en Caen (Normandía). En este encuentro se fraguó la intención de formar una Asociación Internacional de Praxiología Motriz. Cuando estábamos comiendo una docena de personas empezamos a comentar esta necesidad de nuestra comunidad científica. Pierre parecía estar entusiasmado con este proyecto, cogió un papel y un lápiz y escribió las siglas AIPRAM, valía para español o para francés, perfecto, dijo.

Al año siguiente, en la universidad de La Plata, República Argentina, con motivo de un tercer seminario sudamericano, se celebró la primera asamblea de la reciente creada asociación, era el mes de octubre de 2011, y allí se eligió la primera Junta Directiva de esta asociación internacional de carácter científico.

AIPRAM nació y creció con entusiasmo organizando el primer congreso en Manaus (Brasil) en 2014, en donde los colegas brasileños, encabezados por Marco Bortoleto, empezaban a preparar la investidura de Pierre Parlebas como doctor honoris en la universidad de Campinas en 2015. después vino el II Congreso en Chlef (Argelia) en 2016 y finalmente el III Congreso en París (Francia) en verano de 2018.

Sin embargo, AIPRAM parece perdiendo pulso e iniciativas. Se necesita renovar la Junta Directiva con una profunda inyección de investigadores y docentes jóvenes que aporten nuevas iniciativas para estimular a nuevas generaciones, como los nuevos doctores que participan activamente con el grupo de Lleida, conocido ahora como GIAM.

Los jubilados ya somos historia, salvo el gran maestro que sigue fresco y lozano. Léase el último artículo que publicó en enero de 2018 en www.accionmotriz.com "Una pedagogía de las competencias motrices", un texto, 
claro y conciso que ilumina la educación de las conductas motrices como un nuevo paradigma del siglo XXI.

Tenemos una gran oportunidad que nos regala la vida para tener entre nosotros al gran maestro, tan clarividente y preciso. Tendremos que motivar la renovación de AIPRAM para popularizar en este siglo el paradigma de la educación de las conductas motrices, que nos llega desde hace cincuenta años y sigue siendo minoritario, tan necesario como lo sea el de la sostenibilidad, además compatibles ambos, que van a ayudar a transformar la historia de la civilización humana.

La vida me cerró las luces del lenguaje a finales del año 2017 y me ha dejado aparcado como jubilado, pero a la vez, con la tranquilidad necesaria para degustar el maravilloso regalo que nos hizo el amigo Raúl Martínez de Santos, con la publicación en español de los artículos más representativos del genio de Parlebas.

Gracias Raúl y Pierre por este magnífico regalo de "La aventura praxiológica...".

Este retiro necesario, por mis limitaciones actuales, me ha permitido gozar de la producción monumental del genio de Pierre. No tenemos nada que inventar, tan solo tendremos que leer, comprender y aplicar a Parlebas. Esta es nuestra misión actual, urgente y necesaria. Todo está escrito por Pierre desde 1967, hay que aplicarlo.

Las necesidades de las exigencias de la realidad me obligaron a comenzar por intentar divulgar lo más difícil, las investigaciones que aportaban evidencias científicas para que no siguieran acusándolo de encantador de serpientes. Algo también nos ocurrió en el grupo de investigador en Lleida, ahora GIAM, presionados por la necesidad de investigar, tuvimos que aparcar la necesidad de divulgar con claridad y sencillez las ventajas del nuevo paradigma de las conductas motrices.

Necesitamos llegar hasta el hueso de la praxiología motriz, para ello tenemos que llegar a todos los profesores de educación física del mundo. Por supuesto que también debemos dirigirnos a todos los estudiantes de primer curso de educación física. Aquí tenemos aún una fuerte oposición, por la fuerza que sigue teniendo el viejo paradigma del movimiento, relegado en las ciencias a la física mecánica y sobrepasada por la relatividad, la termodinámica, la física atómica o la teoría cuántica.

Tendremos que explicar claramente en qué consiste una conducta motriz, una construcción teórica, una abstracción que termina, por definición, de las dualidades de la realidad humana, que vienen desde el "res cogitans" y del "res 
extensa" de Descartes, desde la Ilustración.

Por esto acabaría con el siguiente corolario para brindarlo a las nuevas generaciones de jóvenes que espero pueblen nuestra comunidad científica internacional:

1) La conducta motriz es única y personal, siempre conduce a la persona, hacía su unidad: física, energética, emocional y afectiva, todo va junto. Está muy bien explicado en los textos de Pierre.

2) Tendremos que explicar que las conductas motrices suponen aceptar un nuevo paradigma, no tan solo para hacer gimnasia, para asistir a una clase de educación física. Y también para caminar por la calle, sentarse o levantarse de una silla o pararse de pie frente un semáforo. Igualmente para vivir, para dar sentido a la vida, gozarla y sentir como se adapta a cualquier situación motriz, cualquiera que sea la edad y la condición física de persona.

3) Tendremos que explicar y aplicar, con claridad y contundencia, las conductas motrices están repletas de afectividad. Dice Parlebas que la afectividad es la llave de las conductas motrices, tanto que sin la afectividad no puede interpretarse ninguna conducta motriz.

4) Tendremos que experimentar que, aunque las conductas motrices pueden vivir inconscientes, se vive a ciegas sin ellas, puede entrenarse la conciencia de las conductas motrices y este proceso, que no es muy complejo, supone descubrir un auténtico tesoro para cada persona, algo que viene junto a los huesos, músculos y órganos, la introyección de las conductas motrices es el "abc" de una nueva educación general liberadora.

5) Tendremos que estimular la creatividad con las conductas motrices, no se trata de repetirlas, de imitarlas, esto es casi imposible. Nada se repite aunque se piense, esto es una creencia errónea, la creatividad le conduce a la persona a fluir con su motricidad, con sus conductas motrices conscientes.

6) Tendremos que conocer y explicar que las conductas motrices son una fuente de empatía, con las propias necesidades, en la comunicación y relaciones con los demás. Un mecanismo de interacción que nos proporciona la llave que abre a la sociedad a transformarse hacia una vida pacífica, tranquila, cooperativa y compasiva.

7) Tendremos que leer, comprender y aplicar que las conductas motrices están evolucionando cada día, para un futbolista, para una peluquera, para un policía, para una enfermera, para una atleta... Las conductas motrices pueden evolucionar optimándose hasta el infinito, hacia fuera y hacia adentro, porque siempre se adapta lo mejor posible a cualquier situación motriz en que se viva o 
se participe. Optimizar y mejorar la calidad de las conductas motrices es posible hasta el final de los días conscientes.

8) Las conductas motrices siempre están interaccionando con el entorno, con uno mismo, con tu propio tono vital, tu ánimo, tu afectividad o tus emociones, e interaccionando y relacionándose con las conductas motrices de los demás.

Tenemos mucho trabajo y mucha responsabilidad para divulgar este legado monumental que nos deja este genial sabio llamado Pierre Parlebas.

Gracias a la vida que nos ha regalado esta gran persona y magnífico personaje que lega a la historia de la civilización humana un auténtico tesoro.

\section{Pere lavega:}

Finaliza aquí esta entrevista. Un auténtico tesoro para los profesionales de la educación física y los amantes de la ciencia de la acción motriz. Muchas gracias amigo Paco. 